\title{
Magnetization, accretion, and outflows in young stellar objects
}

\author{
Frank H. Shu ${ }^{1}$, Daniele Galli ${ }^{2}$, Susana Lizano ${ }^{3}$, Mike J. Cai ${ }^{4}$ \\ ${ }^{1}$ Department of Physics, University of California, San Diego, CA 92093 \\ ${ }^{2}$ INAF-Osservatorio Astrofisico di Arcetri, Largo E. Fermi 5, Firenze I-50125, Italy \\ ${ }^{3}$ CRyA, Universidad Nacional Autónoma de México, Apdo. Postal 72-3, 58089 Morelia, Mexico \\ ${ }^{4}$ Academia Sinica, Institute of Astronomy and Astrophysics, Taiwan
}

\begin{abstract}
We review the theory of the formation and gravitational collapse of magnetized molecular cloud cores, leading to the birth of $\mathrm{T}$ Tauri stars surrounded by quasi-Keplerian disks whose accretion is driven by the magnetorotational instability (MRI). Some loss of magnetic flux during the collapse results typically in a dimensionless mass-to-flux ratio for the star plus disk of $\lambda_{0} \approx 4$. Most of the mass ends up in the star, while almost all of the flux and the angular momentum ends up in the disk; therefore, a known mass for the central star implies a computable flux in the surrounding disk. A self-contained theory of the MRI that drives the viscous/resistive spreading in such circumstances then yields the disk radius needed to contain the flux trapped in the disk as a function of the age $t$. This theory yields analytic predictions of the distributions with distance $\varpi$ from the central star of the surface density $\Sigma(\varpi)$, the vertical magnetic field $B_{z}(\varpi)$, and the (sub-Keplerian) angular rotation rate $\Omega(\varpi)$. We discuss the implications of this picture for disk-winds, X-winds, and funnel flows, and we summarize the global situation by giving the energy and angular-momentum budget for the overall problem.
\end{abstract}

Keywords. Accretion disks; MHD; stars: formation, magnetic fields; ISM: jets and outflows.

\section{Introduction}

Contemporary star formation occurs in dense cores found in giant molecular clouds (Shu et al. 1987; Evans 1999). Two viable but divergent opinions hold concerning the mechanism that produces such cores. The first relies on the leakage of magnetic support by the action of ambipolar diffusion (e.g., Mestel \& Spitzer 1956; Nakano 1979; Lizano \& Shu 1989; Basu \& Mouschovias 1994). The second invokes transient compression by converging turbulent flows (e.g., Elmegreen 1993; Padoan 1995; Klessen, Heitsch, \& MacLow 2001; Vázquez-Semadeni 2005). Recent observations show that early-stage, loosely aggregated, cores in the Pipe Nebula (Lada et al. 2007) and late-stage, tightly aggregated, cores in the Rho Oph region (André et al. 2007) are both internally quiet and have little core-to-core relative motion. These facts demonstrate that hypersonic turbulence of the variety embraced by the early enthusiasts plays little role in the evolution of dense cores into stars and planetary systems. Turbulence may yet enter in initiating core formation through its decay or in triggering the formation of the most massive stars under very crowded conditions, but these possibilities are not of interest in the present review.

Many studies, conducted under a variety of assumptions and boundary conditions, show that the end product of the leakage of magnetic fields from a slowly condensing, lightly ionized, dense pocket of gas and dust by ambipolar diffusion is a gravomagneto catastrophe, whereby the central regions of the condensing core formally tries to reach infinite density in finite time. A useful convention is to define $t=0$ as the moment of catastrophe, with $t<0$ being the condensation phase driving starless cores toward gravomagneto catastrophe, and $t>0$ being the subsequent, inside-out, dynamical collapse to 
form a star plus disk. In a spherical coordinate system $(r, \theta, \varphi)$ with origin at the core center, a nonlinear attractor involving magnetized isothermal support against self-gravity apparently exists that produces solutions in which the density $\rho$ and magnetic flux function $\Phi$ at the critical instant $t=0$ approach the form of a singular isothermal toroid (Li \& Shu 1996):

$$
\rho \rightarrow \frac{a^{2}}{2 \pi G r^{2}} R(\theta), \quad \Phi(r, \theta) \rightarrow \frac{4 \pi a^{2} r}{G^{1 / 2}} \phi(\theta),
$$

where $a$ is the isothermal sound speed and $R(\theta)$ and $\phi(\theta)$ are well-defined functions of the polar angle $\theta$ once the dimensionless mass-to-flux ratio $\lambda$ along flux tubes achieves a critical constant value $\lambda_{0}$ for the inner regions of the core. The likely value of $\lambda_{0}$ varies from 2 to 1 for regions of average to high ionization-fraction (perhaps characteristic of regions of low- and high-mass star formation; see Crutcher \& Troland 2006).

The subsequent evolution for $t>0$ yields a mass infall-rate onto a growing central protostar plus disk that has the form,

$$
\dot{M}=\frac{m_{0}\left(\Theta^{1 / 2} a\right)^{3}}{\left(1-\lambda_{0}^{-2}\right) G},
$$

where $m_{0}$ is a dimensionless coefficient of order unity and $\Theta \geqslant 1$ is a factor accounting for the enhancement of the effective isothermal sound speed $a$ squared associated with the effects of magnetic pressure and possibly turbulence. The natural development of headstart velocities (see Lee et al. 2001; Harvey et al. 2002) and over-densities (compared to the static singular isothermal sphere; see Harvey et al. 2001) from the previous epoch of ambipolar diffusion yield typical values of $m_{0}$ that are a factor of 2 or 3 larger than the classical value $m_{0}=0.975$ (Shu 1977, Adams \& Shu 2007). For conditions measured in dense molecular cloud cores, $\dot{M}$ might vary from a few times $10^{-6} M_{\odot} \mathrm{yr}^{-1}$ (low-mass star formation) to $\sim 10^{-4}-10^{-3} M_{\odot} \mathrm{yr}^{-1}$ (high-mass star formation).

\section{Catastrophic magnetic braking if field freezing applies}

Because the dynamical collapse occurs quickly compared with ambipolar diffusion, field freezing holds as a rough approximation for the epoch $t>0$. Numerical calculations of the collapse phase using the ZEUS-2D code (Stone \& Norman 1992) reveals difficulties, however, some that were anticipated, and others, not (Allen, Li \& Shu 2003). An anticipated difficulty is that if gravitational collapse occurs to a virtual point because of the absence of rotation, then the central star would end with a mass-to-flux ratio given approximately by $2 \pi G^{1 / 2} M_{*} / \Phi_{*} \sim \lambda_{0} \sim 2$. For a star with mass $M_{*} \sim 1 M_{\odot}$ and radius $R_{*} \sim 3 R_{\odot}$, this would imply a surface field $B_{*} \sim \Phi_{*} / \pi R_{*}^{2} \sim 10^{7} \mathrm{G}$, about four orders of magnitude larger than measured fields on the surfaces of $\mathrm{T}$ Tauri stars. Instead of being dragged in from the interstellar medium, the observed $\mathrm{kG}$ fields in these stars probably result from dynamo action.

The above difficulty was already known to Mestel \& Spitzer (1956) and prompted them to propose that ambipolar diffusion would prevent the discrepancy: the interstellar flux would be left behind in the interstellar medium during the collapse to form a star. The modern situation reviewed above shows that $\lambda_{0}$ at the end of the ambipolar diffusion epoch typically amounts to only 2 , and not $10^{4}$ times larger.

From observations we now know that stars of all masses form via the intermediary of an accretion disk (for recent evidence concerning high-mass stars, see Rodríguez, Zapata \& Ho 2007). Accretion disks have cross-sectional areas that are much larger than stars, so they can easily contain the same flux without having absurdly high levels of magnetic 
field. However, an unexpected surprise in the simulations of Allen, Li \& Shu (2003) was that accretion disks do not form if $\lambda_{0}$ is anything like 2 and field freezing were to apply. Galli et al. (2006) give an analytical demonstration that the magnetic braking associated with the collapse to a central point, which produces a magnetic configuration that is a so-called "split monopole," transfers angular momentum away from the central regions so efficiently by the radiation of torsional Alfvén waves that Keplerian disks cannot form. High-resolution numerical simulations conducted by Fromang et al. (2006) and Price \& Bate (2007) have confirmed these results.

Fortunately, a little bit of flux slippage, perhaps due to electrical resistivity rather than ambipolar diffusion, can go a long way toward promoting disk formation (Shu et al. 2006). From a combination of theory and observations (see Girart et al. 2006), Shu et al. (2007) argue that an additional loss of flux by a factor of 2 on the way down to forming a star plus disk is plausible, yielding the estimate $\lambda_{0} \approx 4$ for the value appropriate for such configurations. Using different starting configurations, Hennebelle \& Fromang (2007) find that disk formation is possible even with field freezing if $\lambda_{0}=20$, but not if $\lambda_{0}=5$. Below, we adopt a value $\lambda_{0}=4$ to compute the flux trapped in the circumstellar disks of YSOs, but an uncertainty of a factor of a few exists in this estimate.

\section{Mean-field MHD of accretion disks}

A net magnetization of the circumstellar disks surrounding YSOs makes the MRI (Hawley \& Balbus 1991; Balbus \& Hawley 1998) a natural candidate for the mechanism of inward transport of matter and outward transport of angular momentum. Such a process is needed to explain why objects like the mature solar system have almost all the mass in the central stars and almost all the angular momentum in the companions (planets) that supposedly formed out of their nebular disks. Unfortunately, no previous MRI simulation is both global and has nonzero net flux threading the disk, perhaps explaining why most MRI simulations give too low an effective viscosity compared to the requirements of real systems (King, Pringle \& Livio 2007; Fromang \& Papaloizou 2007).

\subsection{Turbulent viscosity}

In the absence of relevant numerical simulations, we use mixing length theory to estimate the transport coefficients associated with the turbulence induced by the MRI. Consider the schematic diagram in Figure 1, adapted from Shu et al. (2007). We imagine that accretion in the cylindrically radial direction $\varpi$ stretches the field lines that would otherwise stick vertically through the disk so that the poloidal $\mathbf{B}$ has not only a vertical component $B_{z}$, but also a radial component that reverses sign from $B_{\varpi}^{-}<0$ at the lower surface to $B_{\varpi}^{+}>0$ at the upper surface. We then suppose that turbulent fluctuations occur that occasionally create a loop that pinches and disconnects from its parent field line (see steps 2 and 3 of top panel). Because a detached loop is easy to shear, differential rotation would stretch the radial fluctuation $\delta B_{\varpi}$ into a toroidal field $\delta B_{\varphi}$, where the two fields are related (including the proper sign) by the order of magnitude estimate,

$$
\delta u \delta B_{\varphi} \sim \delta B_{\varpi} \varpi \frac{\partial \Omega}{\partial \varpi} \delta \varpi .
$$

For the fluctuations most effective in transport, the velocity perturbation $\delta u$ is related to the mixing length $\delta \varpi$ by the natural inverse correlation time, $\Omega$,

$$
\delta u \sim \Omega \delta \varpi .
$$




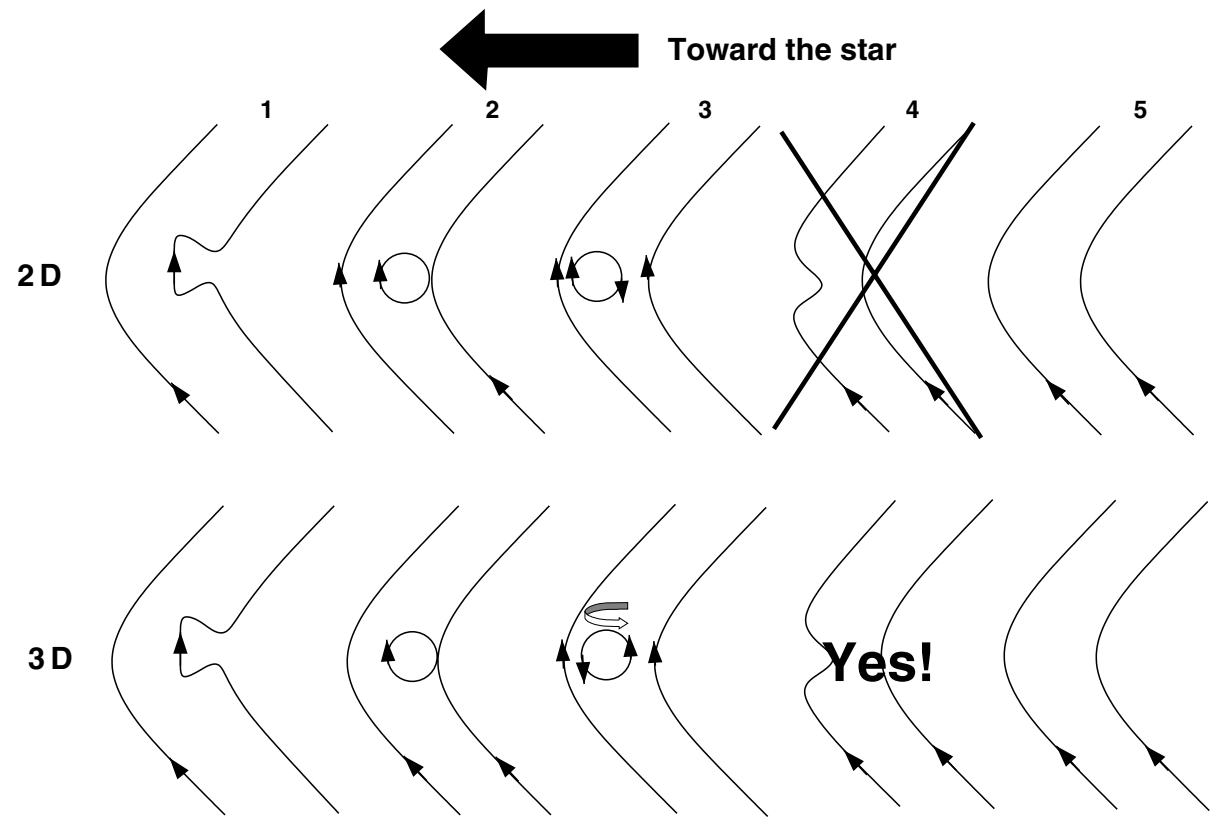

Figure 1. Schematic diagram of scenarios by which field loops are created and destroyed by magnetohydrodynamic turbulence when the mean field is strong: (top) in 2-D by stretch, pinch, disconnect and (bottom) in 3-D by stretch, pinch, disconnect and twist, reconnect, relax. The depiction is the meridional plane $(\varpi, z)$, except for the twist associated with differential rotation indicated by the block arrow, which occurs out of the plane of the page. The twist in the bottom diagram gets the fields oriented in opposite directions at the target reconnection point, which results in the "yes" sign to proceed to steps 4 and 5. (adapted from Shu et al. 2007)

Solving for $\delta B_{\varphi}$, we get

$$
\delta B_{\varphi} \sim \frac{\varpi}{\Omega} \frac{\partial \Omega}{\partial \varpi} \delta B_{\varpi}
$$

Using $B_{\varpi}^{+}$as the natural scale for $\delta B_{\varpi}$, we estimate the associated Maxwell stress as

$$
\frac{\delta B_{\varpi} \delta B_{\varphi}}{4 \pi} \sim \frac{\left(B_{\varpi}^{+}\right)^{2}}{4 \pi} \frac{\varpi}{\Omega} \frac{\partial \Omega}{\partial \varpi} .
$$

The above expression should be compared with the tangential momentum-transport term usually modeled as a viscous stress:

$$
\rho \nu \varpi \frac{\partial \Omega}{\partial \varpi} \sim \frac{\Sigma}{2 z_{0}} \nu \varpi \frac{\partial \Omega}{\partial \varpi}
$$

where $\nu$ is the coefficient of (turbulent) kinematic viscosity, $\Sigma$ is the surface density of the disk (gas plus dust), and $z_{0}$ is its effective half-height. Comparing the two expressions, we may identify

$$
\nu=F\left[\frac{\left(B_{\varpi}^{+}\right)^{2} z_{0}}{2 \pi \Sigma \Omega}\right],
$$

where $F$ is a dimensionless "form factor" of order unity (or somewhat smaller) that corrects for the uncertainties in the twiddles and an effective integration over the thickness of the disk.

In a steady accretion disk where $F$ is a spatial constant, the radial component of the field at the upper surface of the disk is related to the vertical component $B_{z}$ in the 
mid-plane by the equation (Shu et al. 2007):

$$
B_{\varpi}^{+}=I_{\ell} B_{z},
$$

where $I_{\ell}$ is an integral that has a well-determined, order-unity, value once the disk flare is specified (i.e., the power-law dependence of $z_{0}$ on $\varpi$, which is related to the index $\ell$ ). The substitution of equation (3.7) into equation (3.6) yields the form:

$$
\nu=D \frac{B_{z}^{2} z_{0}}{2 \pi \Sigma \Omega}
$$

where $D=I_{\ell}^{2} F$ is an adjustable dimensionless parameter of order unity if the MRI is fully developed. Reassuringly, equation (3.8) is the viscosity expression of Shakura \& Sunyaev (1973) if we substitute magnetic pressure in the mid-plane for gas pressure.

\subsection{Turbulent resistivity}

In order for accretion to occur, not only must outward angular-momentum transport occur diffusively at an enhanced rate relative to friction at a molecular level, but so must inward transport of matter occur by crossing stationary mean-field lines faster than the resistive diffusion associated with knocking ions and electrons off field lines by atomic and molecular collisions. Inward matter transport does not happen if loop dynamics involve only the steps indicated in the top series of picturess in Figure 1. The reason follows.

Consider the matter trapped inside the magnetic loop as the latter tries to move radially inward from the position where it disconnected from the upstream mean field line. If only the $2 \mathrm{D}$ degrees of freedom are considered, this loop cannot attach onto the downstream mean field line because its field orientation is parallel to the target reconnection point. Magnetic reconnection will not occur under such circumstances. The field loop in the sequence of the top panel is thus doomed to bounce (random-walk $\equiv$ diffuse) between the mean field lines sandwiching it, until it accidentally, sooner or later, reattaches to its original parent (upstream) field line. Although the stretching of the field line in the azimuthal direction can diffusively transport some angular momentum by the afore-computed Maxwell stresses, no transport of matter across field lines will occur above and beyond what collisions at a microscopic level allow.

Consider the situation, however, if an extra 3D step of twisting the field loop occurs out of the (meridional) plane of the page, as indicated by the block arrow in panel 3 of the bottom sequence of pictures in Figure 1. Such a vortical twist reverses the inner and outer field directions of the original loop, allowing the loop to attach onto the downstream mean field line by magnetic reconnection. In the process, the enclosed matter will have moved from the upstream field line to the downstream field line at a rate that potentially much exceeds molecular diffusion. We may estimate the associated turbulent diffusion coefficient (turbulent resistivity) by the following product of terms:

$$
\eta=F\left(\frac{B_{\varpi}^{+} B_{z} z_{0}}{2 \pi \Sigma \Omega}\right)\left(-\frac{z_{0}}{\Omega} \frac{\partial \Omega}{\partial \varpi}\right) .
$$

The term in the first parenthesis is the same as its bracketed counterpart in equation (3.6) except that one factor of $B_{\varpi}^{+}$has been replaced by $B_{z}$. Viscous transport depends only on there being a $\left(B_{\varpi}^{+}\right)^{2}$, whereas resistive transport via the formation of loops requires the poloidal field to have mean curvature $B_{\varpi}^{+} B_{z}$. As discussed previously, however, the creation of field loops by itself is not enough to give resistive diffusion. The created field loops must also be able to reattach to downstream field lines, and this requires an added step. For loops of typical vertical size $z_{0}$, the fraction of all formed loops that will have the right orientation for the downstream reconnection can be estimated to be 
the ratio of $z_{0}$ to the radial length associated with the angular shear $-\Omega(\partial \Omega / \partial \varpi)^{-1}$. This ratio is the term inside the second parenthesis. Finally, a dimensionless coefficient of order unity exists in front, which must be taken to equal the same $F$ as appears in equation (3.6) if steady disk accretion is to be possible (Shu et al. 2007).

When equation (3.7) holds and when the rotation law of the disk is quasi-Keplerian, $-(\varpi / \Omega)(\partial \Omega / \partial \varpi)=3 / 2$, the turbulent resistivity is smaller than the turbulent viscosity,

$$
\eta=\frac{3 z_{0}}{2 I_{\ell} \varpi} \nu
$$

by a factor $\sim$ the aspect ratio $z_{0} / \varpi \ll 1$ for a thin disk. First found by Lubow, Papaloizou \& Pringle (1994) via a set of arguments motivated by the desire to drive disk winds, this result is surprising if we use a naive picture of turbulent mixing, but it is a natural outcome when viewed via the model of "loop dynamics" depicted in Figure 1.

\section{Steady-state solution}

When substituted into the governing equations for a steady-state, magnetized, disk with accretion rate $\dot{M}_{\mathrm{d}}$, the viscosity and resistivity expressions yield the following solutions for the rotation rate $\Omega$, surface density $\Sigma$, and vertical component of the magnetic field $B_{z}$ as functions of radial position $\varpi$ in a disk surrounding a star of mass $M_{*}$ :

$$
\begin{gathered}
\Omega(\varpi)=f\left(\frac{G M_{*}}{\varpi}\right)^{1 / 2}, \\
\Sigma(\varpi)=\frac{f}{1-f^{2}}\left(\frac{I_{\ell}}{3 \pi D A}\right) \frac{\dot{M}_{\mathrm{d}}}{\left(G M_{*} \varpi\right)^{1 / 2}}, \\
B_{z}(\varpi)=\left(\frac{2 f}{3 D A}\right)^{1 / 2}\left(\frac{G M_{*} \dot{M}_{\mathrm{d}}^{2}}{\varpi^{5}}\right)^{1 / 4},
\end{gathered}
$$

where $f \leqslant 1$ is a constant accounting for the partial support against stellar gravity by the magnetic tension and $A$ is the disk aspect ratio, $A \equiv z_{0} / \varpi$. The above results are derived in an approximation that ignores the self-gravity of the disk relative to the gravity of the star, and that assumes the effects of gas pressure and magnetic pressure are negligible compared with magnetic tension force in modifying the centrifugal balance of the disk.

The disk aspect ratio is computed from the equation for vertical hydrostatic equilibrium, which yields a relationship between $A$ and the square of the thermal speed $a_{\mathrm{m}}^{2} \equiv k T_{\mathrm{m}} / m$ in the mid-plane of the disk of the form (see Appendix C of Shu et al. 2007):

$$
a_{\mathrm{m}}^{2}=\frac{1}{2}\left[I_{\ell}\left(1-f^{2}\right) A+A^{2}\right] \frac{G M_{*}}{\varpi} .
$$

In principle, we should compute the mid-plane temperature $T_{\mathrm{m}}$ for a thin disk from the condition that radiative conduction and thermal convection (if any) carry the energy of viscous and resistive dissipation to the disk's upper and lower surfaces where this heat is radiated away per unit area at some effective black-body rate $\sigma T_{\mathrm{e}}^{4}$. In practice, for quick modeling purposes, we shall assume $A(\varpi)$ to be given typically and empirically as a power-law, which we will take here to be (see, e.g., D'Alessio et al. 1999):

$$
A(\varpi)=0.1\left(\frac{\varpi}{100 \mathrm{AU}}\right)^{1 / 4}
$$

With $1 / 4$ as the power-law exponent, Shu et al. (2007) compute the self-consistent value 
for $I_{\ell}$ to be 1.742. They also argue that the plausible theoretical domain for this exponent ranges from $1 / 8$ to $1 / 2$, implying that the power-law for $\Sigma$ varies from $\Sigma \propto \varpi^{-5 / 8}$ to $\Sigma \propto \varpi^{-1}$, in agreement with the empirical findings of Andrews \& Williams (2007).

In addition, we suppose that we can ascribe a fiducial age $t_{\text {age }}$ to a disk based on the time it would take to completely drain the disk mass $M_{\mathrm{d}}$ at the accretion rate $\dot{M}_{\mathrm{d}}$ :

$$
\int_{0}^{R_{\Phi}} \Sigma(\varpi) 2 \pi \varpi d \varpi \equiv M_{\mathrm{d}}\left(R_{\Phi}\right)=\dot{M}_{\mathrm{d}} t_{\mathrm{age}} .
$$

In the above we have taken the outer radius of the disk to equal the value $R_{\Phi}$ needed to contain the flux $\Phi_{\mathrm{d}}$,

$$
\int_{0}^{R_{\Phi}} B_{z} 2 \pi \varpi d \varpi=\Phi_{\mathrm{d}}=2 \pi G^{1 / 2} M_{*} / \lambda_{0},
$$

where $\lambda_{0}$ is the dimensionless mass-to-flux ratio brought into the system by the gravitational collapse from a molecular cloud core. When the indicated integrals are performed with the integrands given by the steady-state solutions for $\Sigma(\varpi)$ and $B_{z}(\varpi)$, we are implicitly assuming a definite value for the total angular momentum of the system,

$$
\mathcal{J}_{\mathrm{d}}=\int_{0}^{R_{\Phi}} \varpi^{2} \Omega \Sigma 2 \pi \varpi d \varpi .
$$

This value will generally not equal the actual amount. At late times, the actual system will not have enough angular momentum to maintain the system in steady state all the way out to the flux radius $R_{\Phi}$, and the disk will experience time-dependent spreading.

Other problems may also arise, such as dead zones because of insufficient ionization in the central layers (Gammie 1996). Disregarding such difficulties, in steady state, we can write the requirement of centrifugal balance against the joint attraction of the stellar gravity and the magnetic repulsion (due to tension) acting inside the disk as

$$
\varpi \Omega^{2}=\frac{G M_{*}}{\varpi^{2}}-\frac{B_{\varpi}^{+} B_{z}}{2 \pi \Sigma} .
$$

Using equations (3.7), (4.1), (4.2), (4.3), (4.6), and (4.7), we now transform the above requirement to the following formula for the departure from Keplerian rotation:

$$
1-f^{2}=\left(\frac{0.5444}{\lambda_{0}^{2}}\right) \frac{M_{*}}{M_{\mathrm{d}}\left(R_{\Phi}\right)},
$$

where we have specialized the numerical coefficient to the value applicable to the flaring law (4.5). Because the star's mass $M_{*}$ increases as the disk accretion proceeds, whereas the disk's mass $M_{\mathrm{d}}\left(R_{\Phi}\right)$ decreases, equation (4.10) states that the departure from Keplerian rotation, $1-f^{2}$, increases with time. With a fixed total mass and flux for the system, the disk becomes increasingly magnetized with time, leading to a growing role for magnetic repulsion relative to gravitational attraction of the disk.

\subsection{Four astronomical models}

The four columns following the object in Table 1 gives the input parameters when we assume that $\lambda_{0}=4$ in the models for a T Tauri star, a low-mass protostar, an FU Orionis star, and a high-mass protostar. Notice that the input values are ordinary except for the choice $D=10^{-2.5}$ for the T Tauri model, which is to be contrasted with the more natural selection $D=1$ for the other three models. The small value for $D$ is made so that the T Tauri disk would not have spread to ridiculously large radii $R_{\Phi}$, and it can be partially justified physically on the grounds that $\mathrm{T}$ Tauri stars may have dead zones. 
Table 1. Parameters of four model systems

\begin{tabular}{lcccccccc}
\hline Object & $M_{*}$ & $\dot{M}_{\mathrm{d}}$ & $t_{\text {age }}$ & $D$ & $f$ & $R_{\Phi}$ & $M_{\mathrm{d}}\left(R_{\Phi}\right)$ & $\mathcal{J}_{\mathrm{d}}\left(R_{\Phi}\right)$ \\
\hline & $\left(M_{\odot}\right)$ & $\left(M_{\odot} / \mathrm{yr}\right)$ & $(\mathrm{yr})$ & & & $(\mathrm{AU})$ & $\left(M_{\odot}\right)$ & $\left(M_{\odot} \mathrm{AU} \mathrm{km} / \mathrm{s}\right)$ \\
\hline T Tauri star & 0.5 & $1 \times 10^{-8}$ & $3 \times 10^{6}$ & $10^{-2.5}$ & 0.658 & 298 & 0.0300 & 5.12 \\
Low-mass Protostar & 0.5 & $2 \times 10^{-6}$ & $1 \times 10^{5}$ & 1 & 0.957 & 318 & 0.200 & 51.4 \\
FU Ori & 0.5 & $2 \times 10^{-4}$ & $1 \times 10^{2}$ & 1 & 0.386 & 16.5 & 0.0200 & 0.473 \\
High-mass Protostar & 25 & $1 \times 10^{-4}$ & $1 \times 10^{5}$ & 1 & 0.957 & 1,520 & 10.0 & 39,700 \\
\hline
\end{tabular}

The last four columns in Table 1 yield the output parameters of the analytic theory. A detailed discussion of these results can be found in Shu et al. (2007). For here, we merely note that the predicted sub-Keplerianity $f=0.658$ of $\mathrm{T}$ Tauri disks is surprising and extreme. If we were to make a less extreme choice for $\lambda_{0}$, say, $\lambda_{0}=8$, the quantity $1-f^{2}$ in equation (4.10) would decrease by a factor of 4 , and $f$ would equal (a perhaps more acceptable) 0.926 , with magnetic fields a factor of 2 lower than discussed below.

\subsection{Expected magnetic fields}

Figure 2 shows the magnetic field distribution from equation (4.3) when we adopt the parameter choices of Table 1 . The solid line applies to the $M_{*}=0.5 M_{\odot}$ models and the dashed line to the $M_{*}=25 M_{\odot}$ model, with $f$ chosen to be 0.386 for the former (appropriate to FU Ori) and 0.957 for the latter (appropriate to the high-mass protostar). The disk accretion rate $\dot{M}_{\mathrm{d}}$ and stellar mass $M_{*}$ have been scaled according to the formula that labels the vertical axis. The data points come from Donati et al.(2005) for FU Ori, Winnberg et al. (1981) for V1057 Cyg, Hutawarakorn \& Cohen (1999) for G35.2-0.74N, Hutawarakorn et al.(2002) for W75N, and Edris et al.(2005) for IRAS 20126.

In general, the theoretical expectations are in line with the empirical measurements. The agreement is impressive when we realize that the magnetic fields measured from spectropolarimetry and the Zeeman effect in maser emission lines span almost six orders of magnitude in field strength and over four magnitudes in spatial scale. From Figure 2 , however, we see that a vast observational desert exists in the measurements made at the extremes of this range. In this desert, there exists empirically only the meteoritic measurement of roughly $1 \mathrm{G}$ from chondritic materials that come to us presumably from about $3 \mathrm{AU}$ in the asteroid belt (Levy \& Sonett 1978). Fortunately or fortuitously, this measurement also lies on the theoretical line (see the discussion in Shu et al. 2007). Much work awaits observers in the ALMA era to fill in the gaps of Figure 2.

\section{Implications for disk winds}

The expectation that strong magnetization makes the inner parts of accretion disks sub-Keplerian in their rotation rates has important consequences for the feasibility of disk winds. It is well-known that one criterion for a cold, magnetocentrifugally driven, disk wind to arise is that the $\mathbf{B}$ field should emerge from the disk surface at an outward angle of more than $30^{\circ}$ with respect to the vertical direction (Blandford \& Payne 1982). Translated to our language, this requires $B_{\varpi}^{+} / B_{z}>1 / \sqrt{3}$, which is amply satisfied by equation (3.7) where $B_{\varpi}^{+} / B_{z}=I_{\ell}=1.742$.

Magnetocentrifugally driven disk winds need to satisfy a second criterion, namely, that the acoustic speed at the surface $a_{\mathrm{s}}$ must make up for any departure from Keplerian 


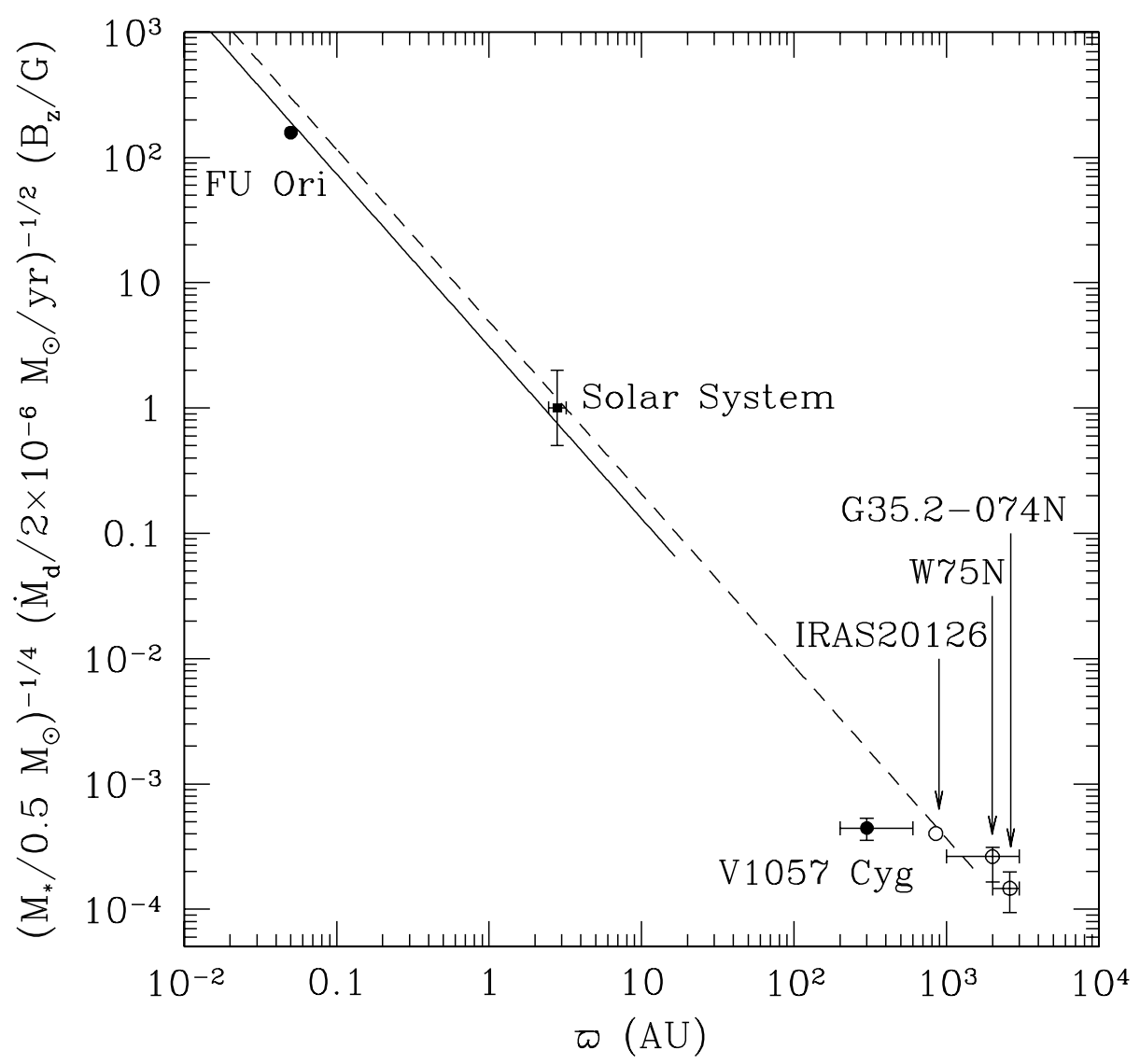

Figure 2. Scaled magnetic field $B_{z}$ plotted against radial distance $\varpi$ in the accretion disk from the central star.

rotation at the launch point $\varpi$, a condition that Shu et al. (2007) express as

$$
a_{\mathrm{s}}^{2}>\frac{1}{4}\left(1-f^{2}\right) \frac{G M_{*}}{\varpi} .
$$

If the departure from Keplerian rotation is zero, $1-f^{2}=0$, then even cold winds with $a_{\mathrm{s}}=0$ can be magnetocentrifugally launched from the disk surface provided that the first criterion $B_{\varpi}^{+} / B_{z}>1 / \sqrt{3}$ is satisfied. However, if $1-f^{2}>0$, then thermal help is needed to overcome the gravitational potential $G M_{*} / \varpi$. The necessary coefficient is estimated as $1 / 4$, because Parker's (1963) theory for the unmagnetized, non-rotating, solar wind produces one factor of $1 / 2$, and another $1 / 2$ can be justified on the grounds that rotation at Kepler speeds brings the gas within a factor of $1 / 2$ of having enough energy to escape.

Comparison with equation (4.4) now reveals the potential problem. The first term in that equation is smaller than the term on the right-hand side of equation (5.1) by the factor $2 I_{\ell} A$, which is a small number in the inner regions of the disk where $A$ has a value of only a few percent. The first term in equation (4.4) dominates in the case of strongly magnetized disks where the vertical thickness of the disk is compressed more by the gradient of the magnetic pressure associated with $B_{\varpi}^{2}$ than it is by the vertical component of the stellar gravity (effect of the second term). But the sound speed in the surface layers cannot exceed the sound speed in the mid-plane, unless the disk surface is heated externally, for example, by high-energy photons (X-rays, UV) coming from the central 


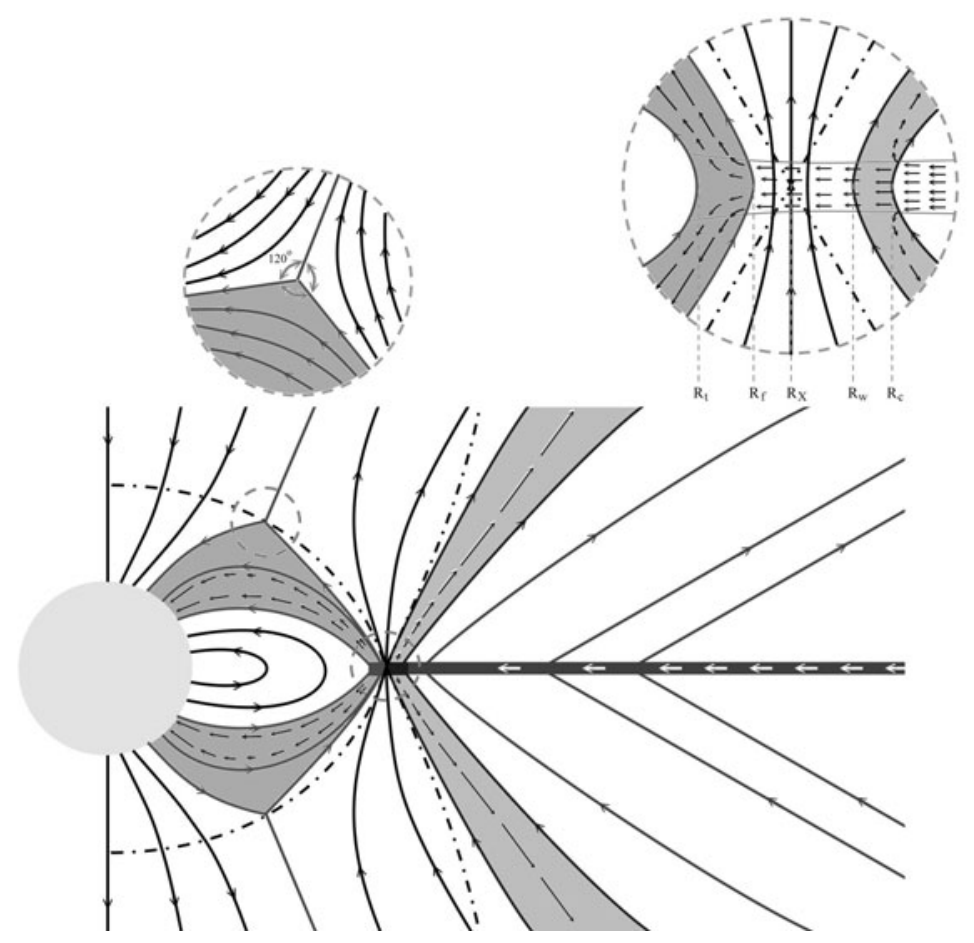

Figure 3. Interaction of a magnetized accretion disk with a strongly magnetized central star.

star. Without external heating, the criterion of equation (5.1) is in contradiction with equation (4.4). Thus, disk winds can be magnetocentrifugally driven in the magnetically dominant regime only if they are warm (cf. the presentation of Cabrit at this conference). In this case the rate-limiting factor in determining the wind mass-loss rate is the stellar photons reaching the disk surface, a condition that would make disk winds closely related to the process of photo-evaporation (e.g., Font et al. 2004). Conversely, if the second term in equation (4.4) dominates, then the magnetization of the disk has to be weak to make $1-f^{2}<2 A^{2}$, severely hampering the ability of the disk to drive powerful, fast, cold jets.

\section{Implications for $\mathrm{X}$-winds}

Fast stellar jets almost certainly require an X-wind (Shu et al. 1994), and only X-winds can make sense of the peculiar interpretation given to the He I observations by Suzan Edwards in her presentation at this conference. The observational results reviewed by Chris Johns-Krull at this conference similarly have no plausible explanation other than $\mathrm{X}$-wind theory. However, why does not sub-Keplerian rotation of the disk have the equally ominous implication for $\mathrm{X}$-winds that it does for disk winds?

\subsection{Influence of disk fields}

Equation (4.9) combined with Figure 3 supplies the answer to the last question. Equation (4.9) states that sub-Keplerian rotation is expected only as long as the product $B_{\varpi}^{+} B_{z}$ is positive, i.e., only when the disk field makes an outward bend. But the sense of the bend must reverse as we come to the inner edge of the disk and the accretion flow attaches onto the stellar magnetosphere in a funnel flow onto the central star (Fig. 3). The reversal in sign of $B_{\varpi}^{+} B_{z}$ as this happens makes the disk rotation rate transition 
from sub-Keplerian values (in the disk proper), to Keplerian values (in the X-region), to super-Keplerian values (in the forbidden region interior to the inner disk edge). Before the latter happens, the disk (dark shade) is truncated and the matter flows onto the star via a magnetospheric funnel (Shu et al. 1994; see also the simulations of Romanova at this conference). The reversed pressure gradients at a sharp inner edge help with making the transition from sub-Keplerian to super-Keplerian values.

To visualize what is going on in Figure 3, imagine the inward press of the disk field to be resisted by the outward press of the squeezed magnetosphere (assumed to have a dipole moment in the picture that is anti-aligned with the disk field; for a more general multipole treatment, see Mohanty \& Shu 2007). The squeezing pops open some of the previously closed stellar fields, and these open fields become available to form the regions that are dead or alive to the magnetocentrifugal flow (field angles at the surface of the disk less than or greater than $30^{\circ}$ from the vertical). The inward press on the stellar magnetosphere also increases the disk rotation rate near the inner edge of the disk. Because of the added presence of the disk fields (with comparable strengths as the compressed stellar field in the X-region), X-winds will be better focused than the case with no disk field. Moreover, the fraction $f_{\mathrm{w}}$ of the disk accretion rate $\dot{M}_{\mathrm{d}}$ in Figure 3 that ends up in outflow (lightly shaded X-wind) rather than inflow (lightly shaded funnel-flow) will be less than $1 / 3$ because of the pushing up of the trapped stellar flux toward a more vertical direction. The lighter loading of field lines will make the terminal speeds of $\mathrm{X}$-winds faster than computed under the assumption that the disk is itself unmagnetized.

\section{2. $X$-winds in action}

The basic equations for steady, axisymmetric, X-winds were written down by Shu et al. (1988) - all that has changed in the interim are the boundary conditions that are attached to the two governing partial-differential equations. For a cold flow, the Grad-Shafranov equation (Grad \& Rubin 1958; Shafranov 1966) for the stream function $\psi(\varpi, z)$ reads:

$$
\nabla \cdot(\mathcal{A} \nabla \psi)+\frac{1}{\mathcal{A}}\left(\frac{J}{\varpi^{2}}-1\right) \frac{J^{\prime}}{\varpi^{2}}+\frac{2 \beta \beta^{\prime} V_{\mathrm{eff}}}{\left(\beta^{2}-\varpi^{2} \mathcal{A}\right)^{2}}=0
$$

where $V_{\text {eff }}=-\left(\varpi^{2}+z^{2}\right)^{-1 / 2}-\varpi^{2} / 2+3 / 2$ is the dimensionless effective potential in the corotating frame. Bernoulli's equation for the Alfvén discriminant $\mathcal{A} \equiv\left(\beta^{2} \rho-1\right) / \varpi^{2} \rho$ is

$$
|\nabla \psi|^{2}+\frac{1}{\mathcal{A}^{2}}\left(\frac{J}{\varpi^{2}}-1\right)^{2}+\frac{2 \varpi^{2} V_{\mathrm{eff}}}{\left(\beta^{2}-\varpi^{2} \mathcal{A}\right)^{2}}=0 .
$$

In the above $J(\psi)$ and $\beta(\psi)$ are functions of $\psi$ associated, respectively, with the conservation of angular momentum carried in matter and field and with the freezing of gas loaded onto the field at the base of the flow from the surface of the disk.

The difficulty with the above formulation is that equation (6.2) substituted into equation (6.1) yields a partial-differential of mixed type, changing from elliptic to hyperbolic as the flow velocity crosses certain signal speeds (Heinemann \& Olbert 1978). In the cold limit, the relevant signal speed is the fast MHD speed, with the Alfvén surface where $\mathcal{A}=0$ being only an apparent singularity where $J(\psi)=\varpi^{2}$. These equations have been attacked in various guises by Najita \& Shu (1994, X-wind in the sub-Alfvénic region), Ostriker \& Shu (1995, funnel flow), Shu et al. (1995, X-wind far asymptotics), and Shang et al. (1998, full X-wind solution by interpolation of near and far asymptotics). This piecemeal treatment has been criticized by other workers (cf. Ferreira et al. 2006), but it has produced global solutions of adequate accuracy for most astronomical applications. 
Nevertheless, a more systematic approach is desirable. Such an approach has been found and implemented by Cai et al. (2007). It begins by defining an action integral:

$$
S \equiv \iint\left[\frac{\mathcal{A}}{2}|\nabla \psi|^{2}-\frac{1}{2 \mathcal{A}}\left(\frac{J}{\varpi^{2}}-1\right)^{2}+\frac{V_{\text {eff }}}{\beta^{2}-\varpi^{2} \mathcal{A}}\right] 2 \pi \varpi d \varpi d z
$$

The action $S$ gives equation (6.1) when extremized with respect to variations of $\psi$, while it gives equation (6.2) when extremized with respect to variations of $\mathcal{A}$. Instead of attacking the two relations as partial differential equations, we may then reduce the problem to looking for trial functions in a variational approach. Details are given in Cai et al. (2007). For here, we merely note that the solutions so obtained are in good agreement with the earlier ones obtained by less sophisticated means.

\section{Budget for energy and angular momentum}

\subsection{Photon Luminosity}

To summarize the outlook of this paper, let us examine the global budget for energy and angular momentum as the gas spirals from large distances in the disk to land in hot-spots at the end of the funnel flow (see also Shu 1995). The hot-spot luminosity is given dimensionally by

$$
L_{\mathrm{hot}}=\left(1-f_{\mathrm{w}}\right) \frac{G M_{*} \dot{M}_{\mathrm{d}}}{R_{*}}\left(1+\frac{R_{*}^{3} \sin ^{2} \theta_{\mathrm{h}}}{2 R_{X}^{3}}-\frac{3 R_{*}}{2 R_{X}}\right),
$$

where $\theta_{\mathrm{h}}$ is the mean co-latitude of the hot-spot. The term inside the parenthesis is $R_{*} / R_{X}$ times the dimensionless specific kinetic energy of gas falling freely from rest at the $\mathrm{X}$-point in the corotating frame,

$$
\frac{1}{2} u^{2}=\frac{1}{r}+\frac{1}{2} \varpi^{2}-\frac{3}{2} \equiv-V_{\mathrm{eff}},
$$

evaluated at the position of the hot-spot on the surface of the star, $r=R_{*} / R_{X}$ and $\varpi=r \sin \theta_{\mathrm{h}}$. To get the system photon-luminosity, $L_{\mathrm{sys}}$, the amount from the hot spot is to be added to the amount $L_{*}$ liberated by the star and the disk-accretion luminosity:

$$
L_{\mathrm{d}}=\left(\mathcal{T}+\frac{1}{2}\right) \frac{G M_{*} \dot{M}_{\mathrm{d}}}{R_{X}},
$$

where $\mathcal{T}$ is the dimensionless viscous torque just outside $R_{X}$. Thus,

$$
L_{\mathrm{sys}}=L_{*}+L_{\mathrm{hot}}+L_{\mathrm{d}} .
$$

\subsection{Flow and Field Luminosity}

In a cold gas, even in the presence of a magnetic field, the dimensionless specific energy of the gas in the corotating frame, $H \equiv|\mathbf{u}|^{2} / 2+V_{\text {eff }}=0$ is a constant of motion. In an inertial frame, $H$ is the Jacobi constant, $H=E^{g}-J^{g}$, where $E^{g}$ and $J^{g}$ are the specific energy and specific angular momentum of the gas measured in an inertial frame, with $E^{g}=1=J^{g}$ at the X-point where $V_{\text {eff }}=0$ by definition. Thus, the time rate of change of the dimensionless specific energy $E^{g}$ of a parcel of gas as we follow its motion is given by the time rate of change $J^{g}$ along a streamline. There is also a Poynting flux per unit mass flux given by the analogous quantity $J^{B}$. Thus, in a convention where we define the gravitational potential energy in an inertial frame to be zero at infinity, the mechanical 
luminosity flowing out of the system in the form of an $\mathrm{X}$-wind is given by

$$
L_{\mathrm{w}}=f_{\mathrm{w}}\left(\bar{J}_{\mathrm{w}}-\frac{3}{2}\right) \frac{G M_{*} \dot{M}_{\mathrm{d}}}{R_{X}}
$$

where $\bar{J}_{\mathrm{w}}$ is the dimensionless specific angular-momentum carried on average in wind gas and field. The constant streamline-averaged quantity $\bar{J}_{\mathrm{w}}=\bar{J}_{\mathrm{w}}^{g}+\bar{J}_{\mathrm{w}}^{B}$ is the sum of two variables where the gaseous contribution $\bar{J}_{\mathrm{w}}^{g}$ starts at 1 at $R_{X}$ and becomes $\bar{J}_{\mathrm{w}}>3 / 2$ at infinity, whereas the field contribution $\bar{J}_{\mathrm{w}}^{B}$ starts at $\bar{J}_{\mathrm{w}}-1$ at $R_{X}$ and goes to zero at infinity. Similarly, the mechanical luminosity contained in the funnel flow equals

$$
L_{\text {fun }}=\left(1-f_{\mathrm{w}}\right)\left(\bar{J}_{*}-\frac{3}{2}\right) \frac{G M_{*} \dot{M}_{\mathrm{d}}}{R_{X}} .
$$

The constant streamline-averaged quantity $\bar{J}_{*}=\bar{J}_{*}^{g}+\bar{J}_{*}^{B}$ is the sum of two variables where the gaseous contribution $\bar{J}_{*}^{g}$ starts at 1 at $R_{X}$ and becomes $\bar{J}_{*}$ at the hot-spot, whereas the field contribution $\bar{J}_{*}^{B}$ starts at $\bar{J}_{*}-1$ at $R_{X}$ and goes to zero at the hot spot (if the hot-spot field is very strong).

If we add the two mechanical luminosities to the power dissipated in the disk to accrete the gas from infinity to $R_{X}$, we get

$$
L_{\mathrm{w}}+L_{\text {fun }}+L_{\mathrm{d}}=\frac{G M_{*} \dot{M}_{\mathrm{d}}}{R_{X}}\left[f_{\mathrm{w}} \bar{J}_{\mathrm{w}}+\left(1-f_{\mathrm{w}}\right) \bar{J}_{*}-1+\mathcal{T}\right],
$$

which must equal zero since the gas and field in the star formation process starts with zero specific energy at infinity and energy is conserved. This requires the expression in the square bracket to vanish, which is equivalent to the conservation of angular momentum because what leaves the X-region in the X-wind, $f_{\mathrm{w}} \bar{J}_{\mathrm{w}}$, and in the funnel flow, $\left(1-f_{\mathrm{w}}\right) \bar{J}_{*}$, must come from what was originally there, 1 , minus any angular momentum per unit mass removed by the viscous torque, $\mathcal{T}$. The conservation of energy is equivalent to the conservation of angular momentum because, by the argument involving the Jacobi integral, the only way to transfer energy (in the inertial frame) is to exert torque. In any case, the conservation of angular momentum/energy in the X-region requires

$$
f_{\mathrm{w}}=\frac{1-\mathcal{T}-\bar{J}_{*}}{\bar{J}_{\mathrm{w}}-\bar{J}_{*}} .
$$

The fact that the sum of the three luminosities,

$$
L_{\text {fun }}+L_{\mathrm{w}}+L_{\mathrm{d}}=0,
$$

vanishes means that $L_{\text {fun }}$ must be negative if $L_{\mathrm{w}}$ and $L_{\mathrm{d}}$ are positive. Mechanical power flows out of the star because it gives up its right to rotate faster and faster if it becomes locked to the rotation rate of the inner edge of the disk. Although this happens formally for arbitrary $\mathcal{T}$ and $\bar{J}_{*}$ as long as $\bar{J}_{*}<3 / 2$ and $\mathcal{T}<1-\bar{J}_{*}$; in fact we expect $\bar{J}_{*}$ and $\mathcal{T}$ to be small compared to unity if the square of the stellar radius is small in comparison to $R_{X}^{2}$ and if the MRI torque just outside $R_{X}$ cannot compete with wind or funnel flows that remove or add angular momentum at dynamical rates.

The physical significance of $L_{\text {fun }}$ being negative cannot be overstated. Angular momentum is transferred by the funnel flow to the inner edge of the disk, except for a small part that is needed to keep the star locked to the disk. Otherwise, the star would begin to rotate faster and faster, becoming highly flattened as a result. Disk truncation occurs not by ram pressure effects (Ghosh \& Lamb 1978), but by considerations of angularmomentum transport. Funnel flows are the reason why stars are spheres and not disks. 
This fact is the ultimate tribute to the proposal made by Claude Bertout and Gibor Basri many years ago that magnetospheric infall underlies the accretion luminosity of $\mathrm{T}$ Tauri stars, not boundary-layer emission.

\section{References}

Adams, F. C., \& Shu, F. H. 2007, ApJ, submitted

Allen, A, Li, Z.-Y., \& Shu, F. H. 2003, ApJ, 599, 363

André, P., Belloche, A., Motte, F., \& Peretto, N. 2007, A\&A, in press

Andrews, S. M., \& Williams, J. P. 2007, ApJ, 659, 705

Balbus, S. A., \& Hawley, J. F. 1998, Rev. Mod. Phys., 70, 1

Basu, S., \& Mouschovias, T. Ch., 1994, ApJ, 432, 720

Blandford, R. D., \& Payne, D. G. 1982, MNRAS, 199, 883

Cai, M. J., Shang, H., Lin, H.-H., \& Shu, F. H. 2007, ApJ, submitted

Crutcher, R. M., \& Troland, T. H. 2006, in Triggered Star Formation in a Turbulent ISM, IAU Symp. No. 237, 25

D'Alessio, P., Calvet, N., Hartmann, L., Lizano, S., \& Canto, J. 1999, 527, 893

Donati, J. F., Paletou, F., Bouvier, J., \& Ferreira, J. 2005, Nature, 438, 466

Edris, K. A., Fuller, G. A., Cohen, R. J., \& Etoka, S. 2005, A\&A, 434, 213

Elmegreen, B. G. 1993, ApJL, 419, L29

Evans, N. J. 1999, ARAA, 37, 311

Ferreira, J., Dougados, C., \& Cabrit, S. 2006, A\&A, 453, 785

Font, A. S., McCarthy, I. G., Johnstone, D., \& Ballantyne, D. R. 2004, ApJ, 607, 890

Fromang, S., Hennebelle, P., \& Teyssier, R. 2006, A\&A, 457, 371

Fromang, S., \& Papaloizou, J. 2007, Astro-ph 0705.3621

Galli, D., Lizano, S., Shu, F. H., \& Allen, A. 2006, ApJ, 647, 374

Gammie, C. F. 1996, ApJ, 457, 355

Ghosh, P., \& Lamb, F. K. 1978, ApJ, 223, L83

Girart, J. M., Rao, R., \& Marrone, D. 2006, Science, 313, 812

Grad, H., \& Rubin, H. 1958, in Proc. Conf. Internat. Atomic Energy Agency 31, (Geneva: Internat. Atomic Energy Agency)

Harvey, D.W.A., Wilner, D.J., Lada, C.J., Myers, P.C., Alves, J.F., \& Chen, H. 2001, ApJ, 563, 903

Harvey, D.W.A., Wilner, D. J., DiFrancesco, J., Lee, C. W., Myers, P. C., \& Williams, J. P. 2002, AJ, 123, 3325

Hawley, J. F., \& Balbus, S. A. 1991, ApJ, 381, 496

Heinemann, M., \& Olbert, S. 1978, JGR, 83, 2457

Hennebelle, P., \& Fromang, S. 2007, A\&A, submitted

Hutawarakorn, B., \& Cohen, R. J. 1999, MNRAS, 303, 845

Hutawarakorn, B., Cohen, R. J., \& Brebner, G. C. 2002, MNRAS, 330, 349

King, A. R., Pringle, J. E., \& Livio, M. 2007, MNRAS, 376, 1740

Klessen, R. S., Heitsch, F., \& MacLow, M. 2001, ApJ, 535, 887

Lada, C. J., Muensch, A. A., Rathborne, J., Alves, J. F., \& Lombardi, M. 2007, ApJ, submitted

Lee, C. W., Myers, P. C., \& Tafalla, M. 2001, ApJS, 136, 703

Levy, E. H., \& Sonett, C. P. 1978, in Protostars \& Planets, ed. T. Gehrels (Tucson: University of Arizona Press), p. 516

Li, Z.-Y., \& Shu, F. H. 1996, ApJ, 472, 211

Lizano, S., \& Shu, F. H. 1989, ApJ, 342, 834

Lubow, S. H., Papaloizou, J. C. B., \& Pringle, J. E. 1994, MNRAS, 267, 235

Mestel, L., \& Spitzer, L.1956, MNRAS, 116, 503

Mohanty, S., \& Shu, F. H. 2007, ApJ, in preparation

Najita, J. R., \& Shu, F. H. 1994, ApJ, 429, 808

Nakano, T. 1979, PASJ, 31, 697

Ostriker, E. C., \& Shu, F. H. 1995, ApJ, 447, 813 
Padoan, P. 1995, MNRAS, 277, 377

Parker, E. N. 1963, Interplanetary Dynamical Processes (New York: Interscience Pub)

Price, D. J., \& Bate, M.R. 2007, MNRAS, 337, 77

Rodríguez, L. F., Zapata, L. A., \& Ho, P. T. P. 2007, ApJL, L143

Shafranov, V. D. 1966, Rev. Plasma Phys, 2, 103

Shakura, N. I., \& Sunyaev, R. A. 1973, A\&A, 24, 337

Shu, F. H. 1977, ApJ, 214, 488

Shang, H., Shu, F. H., \& Glassgold, A. E. 1998, ApJ, 439, 91

Shu, F. H. 1995, Rev Mex AA, 1, 375

Shu, F. H., Adams, F. C., \& Lizano, S. 1987, ARAA, 25, 23

Shu, F. H., Galli, D., Lizano, S., \& Cai, M. 2006, ApJ, 647, 382

Shu, F. H., Gallli, D., Lizano, S., Glassgold, A. E., \& Diamond, P. 2007, ApJ, 665, 535

Shu, F. H., Lizano, S., Ruden, S. P., \& Najita, J. 1988, ApJ, 328, L19

Shu, F., Najita, J., Ostriker, E., Wilkin, F., Ruden, S., \& Lizano, S. 1994, ApJ, 429, 781

Shu, F. H., Najita, J., Ostriker, E. C., \& Shang, H. 1995 ApJ, 455, L155

Stone, J. M., \& Norman, M. L. 1992, ApJS, 80, 791

Vázquez-Semadeni, E. 2005, in The Initial Mass Function 50 Years Later, eds. E. Corbelli, F. Palla, \& H. Zinnecker (Dordrecht: Springer), p. 371

Winnberg, A., Graham, D., Walmsley, C. M., \& Booth, R. S. 1981, A\&A, 93, 79

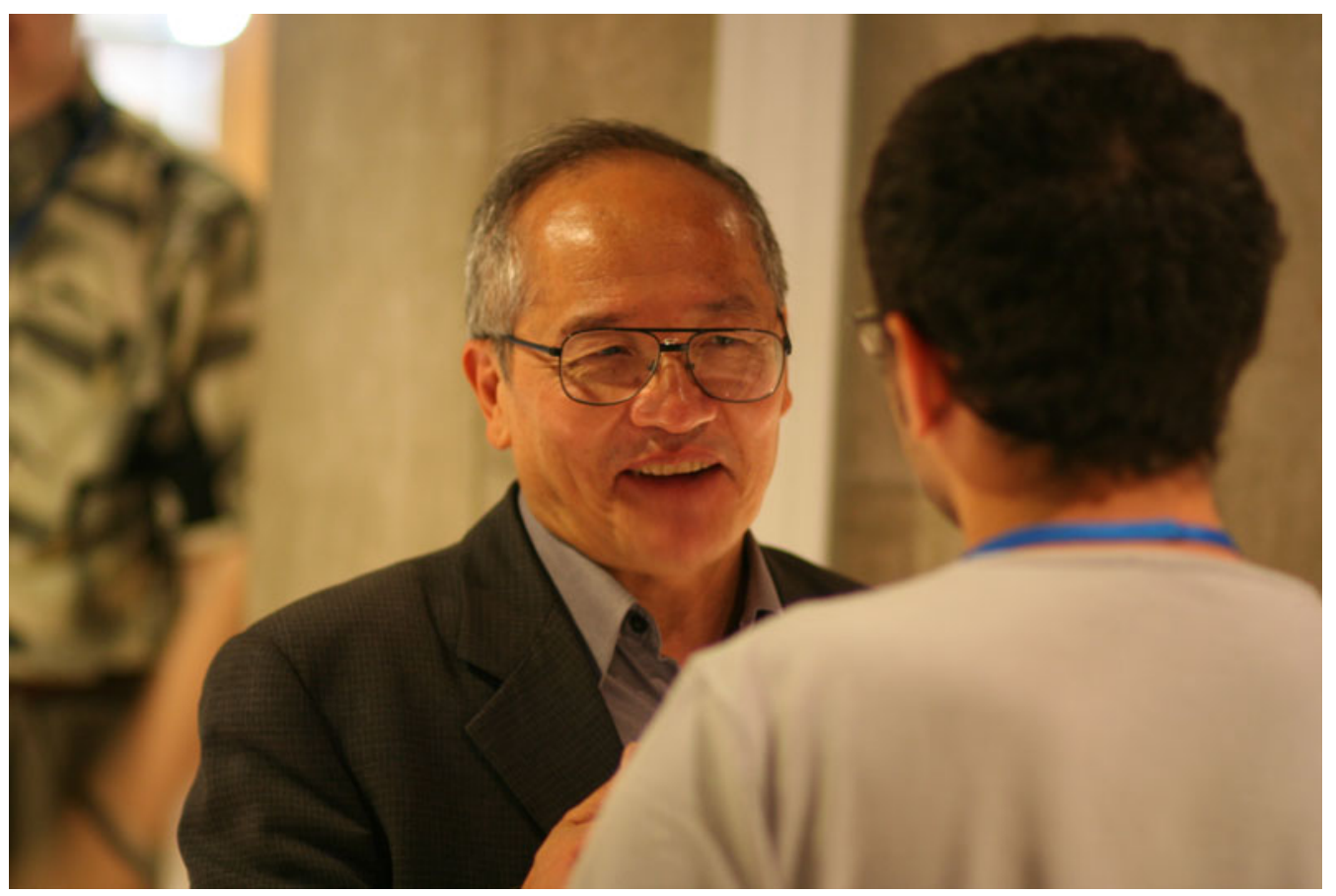



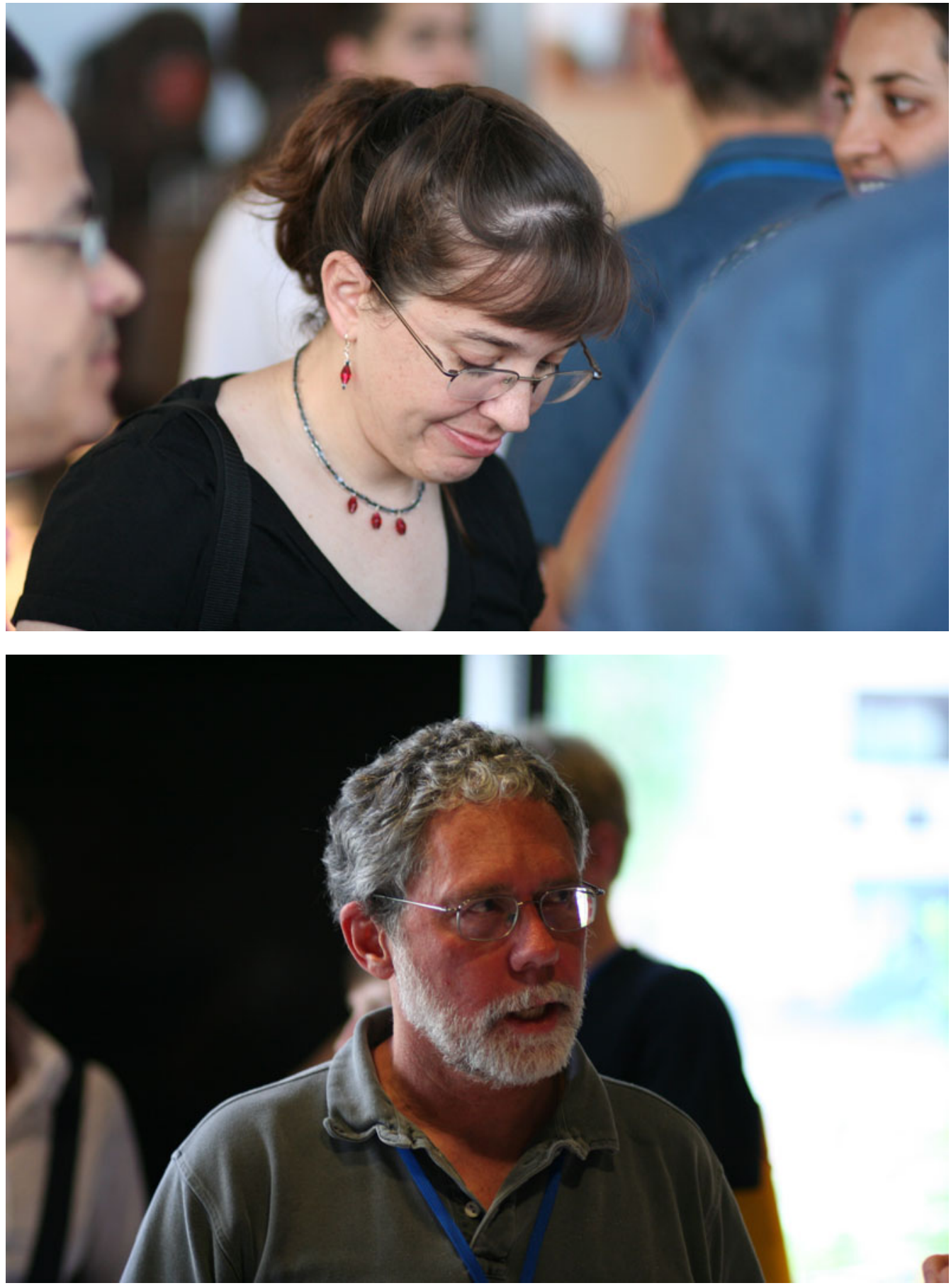